\title{
Trastornos del gusto como indicador de enfermedad sistémica
}

\author{
Taste disorders as an indicator of systemic disease
}

Karla Ivohnne Pedraza Maquera ${ }^{1 a}$ Caroll Johana Uberlinda Lévano ${ }^{1 b}$
https://orcid.org/0000-0002-8156-833X

https://orcid.org/0000-0003-1072-374X

Correspondencia: karlaivho@gmail.com

\section{Resumen}

El objetivo de esta revisión bibliográfica es actualizar las bases teóricas sobre la fisiología del gusto y su importancia en la detección temprana y oportuna de diversas enfermedades sistémicas, teniendo en cuenta la forma en que se expresan las células gustativas y su implicancia directa con enfermedades sistémicas o metabólicas como la diabetes mellitus, hipertensión, 2019-nCoV; esta información es de suma importancia en la actualización de la terapéutica médica-odontológica. Resultando fundamental para el cirujano dentista el desarrollo adecuado del interrogatorio y la aplicación de métodos de diagnóstico, además de entender el desarrollo del proceso del sentido del gusto. Para esta revisión, los datos utilizados se encuentran publicados en la base de datos de búsqueda y selección de PubMed, Google Scholar, Elsevier, Scielo y Web of Science y libros sobre el tema.

Palabras clave: trastorno del gusto, papilas gustativas, diabetes mellitus, hipertensión y 2019-nCoV

\begin{abstract}
The objective of this bibliographic review is to update the theoretical bases of taste physiology and its importance in the early and timely detection of various systemic diseases, taking into account the way in which taste cells are expressed and their direct implication with systemic or metabolic diseases such as diabetes mellitus, hypertension, 2019-nCoV. This information is of utmost importance in the updating of medical-dental therapeutics. The proper development of questioning and the application of diagnostic methods is essential for the dental surgeon; it also allows him to understand the development of the sense of taste process. The data used for this review can be found in the PubMed, Google Scholar, Elsevier, Scielo and Web of Science search and selection databases, and books on the subject.
\end{abstract}

Keywords: taste disorder, taste buds, diabetes mellitus, hypertension and 2019-nCoV

\footnotetext{
${ }^{1}$ Investigador independiente. Tacna, Perú

a Cirujano Dentista. Maestra en Odontología con mención en Patología

b Cirujano Dentista. Maestro en Ciencias con mención en Salud Pública
} 


\section{Introducción}

Aproximadamente, cerca de 200000 personas acuden al médico cada año por afecciones en los sentidos del gusto o del olfato. Los investigadores estiman que hasta un $15 \%$ de adultos podrían tener una afección del gusto o del olfato, pero la mayoría no acude a consulta médica. ${ }^{1}$

Los trastornos del gusto pueden llegar a tener un efecto dañino en la vida del ser humano. El sentido del gusto motiva el comer; por lo tanto, tiene una función importante en la nutrición y en la detección de elementos nocivos para el organismo. Científicamente se ha estipulado que existen cinco sabores diferentes que intervienen en el sentido del gusto: amargo, salado, dulce, agrio (ácido) y umami.

La llamada sensopercepción gustativa es primordial para la salud, proporciona mayor capacidad de diferenciar los sustratos que se incorporan al organismo. Esta percepción del sabor requiere fundamentalmente de estructuras denominadas botones gustativos, que se localizan en la cavidad bucal. En la odontología contemporánea se necesita de un análisis del sentido del gusto, considerando los quimiorreceptores, estímulos, mecanismos de transducción y su conectividad compleja; puesto que permite comprender cómo se integran diferentes funciones moleculares que se presentan en el sistema estomatognático, ${ }^{2}$ percibiendo los mecanismos de transducción y las modalidades elementales del gusto. Aportando el conocimiento para afrontar de modo adecuado una problemática de alteración gustativa, identificando las probables causas y adoptar medidas valederas para resolverla. ${ }^{3}$

Las manifestaciones orales pueden preceder a la aparición de ciertas enfermedades sistémicas o acompañarlas en su evolución, de ahí la importancia de conocer estas manifestaciones, tanto por el odontoestomatólogo como por el médico general, para realizar su correcto diagnóstico y tratamiento. ${ }^{2}$

Esta revisión se puntualiza en los causales fundamentales de alteración, reducción y pérdida del gusto; de su posible repercusión en los hábitos alimentarios y la salud; además de la presencia de la pérdida del gusto en diversas enfermedades sistémicas y con especial interés a las hipótesis actualmente desarrolladas sobre los mecanismos por medio de los cuales el 2019-nCoV podría afectar la percepción del gusto. ${ }^{4}$

\section{Fisiología del sentido del gusto}

El sentido del gusto se basa en la interpretación química de los diferentes alimentos que consumimos; de igual forma, el sentido del olfato, en conjunto con el sentido del gusto, nos permiten distinguir, químicamente, alimentos deliciosos, indeseables y hasta mortales. ${ }^{5,6}$ Los sabores dulces, salado, ácido, amargo y umami actúan en conjunto para responder a la función digestiva y mediar respuestas apetito-protectoras, tales como la ingesta de sales o proteínas, si así el organismo lo necesita; ;,7 un ejemplo es el deseo de consumir chocolates por la falta de magnesio en el organismo. Tanto el sentido del gusto como del olfato tienen receptores químicos o quimiorreceptores que son estimulados por moléculas disueltas en la saliva o en el moco nasal; en la lengua, estas moléculas se ponen en contacto con los botones gustativos durante la masticación, y a nivel de las microvellosidades se da lugar a la transducción sensorial. ${ }^{8,7}$

En la lengua hay, aproximadamente, 10 mil papilas gustativas conformadas por cuatro tipos de células: las basales, oscuras, claras e intermedias. Las células basales no se consideran como células del sabor por estar en contacto con la membrana basal y por participar en la diferenciación y recambio de las otras células gustativas, que dura aproximadamente 10 días. ${ }^{6,8}$ Las otras tres células gustativas participan en la fisiología del sabor y también reciben el nombre de células gustativas tipo I, II y III. ${ }^{5-9}$ De acuerdo a su función, los estímulos amargo, dulce y umami son detectados por las células tipo II, ${ }^{5,9}$ siendo que los estímulos amargos 
son detectados, además, por las células tipo III y los estímulos salados ( $\mathrm{NaCl})$, se presume, son detectados por las células tipo I.9,5

Las células gustativas están distribuidas en diferentes porcentajes en la lengua, lo que explica la mayor o menor sensibilidad de la lengua a los diferentes sabores; las células tipo I son aproximadamente el $50 \%$ del total en una papila gustativa, ${ }^{9}$ lo que probablemente explicaría que podamos sentir el sabor salado en casi toda la lengua. Las células tipo II son aproximadamente un tercio de las papilas gustativas, estas funcionan como receptores quimio sensoriales. Las células tipo III son aproximadamente el $15 \%$ del total y se ubican en mayor cantidad en la parte posterior de la lengua, a diferencia de las células tipo II que se ubican en mayor porcentaje en los dos tercios anteriores de la lengua; esto quiere decir que cada papila gustativa contiene los tres tipos de células del sabor, pero en diferentes proporciones.

\section{Mecanismos de percepción del sabor}

Básicamente hay 2 tipos de mecanismos de transducción: Transducción mediada por canales iónicos, la cual permite detectar el sabor salado, ácido y amargo; y transducción mediada por receptores acoplados a proteína G (GPCRS), mecanismo que permite detectar el sabor dulce, amargo y umami. Hay un tercer mecanismo aún no muy claro que es el mediado por el transportador de glucosa tipo 4 (GLUT4), que utiliza el contratransporte con 1 sodio/glucosa (SGLT1) dependiente de $\mathrm{Na}$ (difusión facilitada), ambos transportadores podrían ser una vía alterna de detección de glucosa similar a la utilizada en las células $\beta$ del páncreas. ${ }^{7-9}$

Existen receptores de ATP que no son propiamente del gusto, pero median la transmisión de las células de tipo II a los nervios y la retroalimentación autocrina a las células de tipo II; estos receptores de ATP, serotonina y GABA, son responsables de la comunicación célula-célula, entre las células tipo II y tipo 1 II $^{9}$ y la transmisión serotoninérgica (feedforward) y la transmisión glutamatérgica (feedback) que producen comunicación entre las células de tipo III y las fibras aferentes gustativas. El mecanismo de transducción mediado por canales iónicos es expresado por las células tipo I, que tienen funciones parecidas a las glías; contienen enzimas y transportadores que eliminan los neurotransmisores extracelulares, además de canales iónicos que están asociados a la redistribución y amortiguación del K+.

El sabor salado es detectado por la concentración de $\mathrm{Na}+$ derivado del $\mathrm{NaCl}$ en la saliva, que es captado por las células receptoras a través de los canales de $\mathrm{Na}+$ sensibles a la amilorida $(\alpha \mathrm{ENaC}) \cdot{ }^{7-9} \mathrm{El}$ sodio atraviesa la membrana por medio de los $\alpha \mathrm{ENaC}$ provocando la positivización (despolarización) del medio interno de la célula, provocando la apertura de los canales de calcio por voltaje; los iones $\mathrm{Ca}+$ entran a la célula y, mediante un proceso poco conocido, estimulan a las vesículas sinápticas para que estas liberen el neurotransmisor que rápidamente es captado por las neuronas aferentes gustativas para luego transmitir el mensaje al cerebro. ${ }^{5-8}$ Aunque la percepción del sabor salado aún no está claramente demostrada en humanos, $, 9,10$ estudios en roedores han determinado que la amilorida, un diurético, disminuye la intensidad a la respuesta al $\mathrm{NaCl}$ en los registros de nervios aferentes y reduce la respuesta conductual (lamido) a las soluciones de $\mathrm{NaCl}^{9}$ lo que se corrobora en un estudio efectuado por Charles Zuker et al. (2010) en ratones sin canales $\alpha \mathrm{ENaC}$ en las células gustativas, que resultó en la completa pérdida de la percepción de sal sensible a la amilorida, confirmando que se requiere, al menos, algunos aENaC para la percepción del sabor salado sensible a la amilorida, además, sugirieron que los $\alpha \mathrm{ENaC}$ eran la base del sabor y del apetito por la sal. ${ }^{10}$ Pese a ello se ha tratado de explicar el papel que juega el $\alpha \mathrm{ENaC}$ y el $\mathrm{Na}$ sensible a la amilorida $y$, sobre todo, el papel que juega en el balance de la hipernatremia y deshidratación en seres humanos. ${ }^{9}$ 
La percepción del sabor agrio o ácido se da mediante la concentración de iones $\mathrm{H}$ por acidificación intracelular, siendo los estímulos más potentes el ácido cítrico y el acético; esto es debido a que la membrana es permeable a un ácido orgánico no disociado y a los protones en el citoplasma. ${ }^{9}$

El sabor amargo actúa como medio preventivo ante el consumo de sustancias nocivas para los animales; estas sustancias son percibidas por una variedad de receptores del gusto tipo 2 (T2R) asociadas a proteína G (GPCR). Se considera que los T2R son codificados por 20 o más genes; además de estar presentes en cualquier papila gustativa, lo que le confiere mayor sensibilidad al sabor amargo. ${ }^{2}$

El sabor dulce provoca placer y agrado, este sabor es percibido por receptores de sabor de la familia T1R acoplados a receptores de proteína G; estos receptores específicos son del tipo T1R2 y T1R3, este último receptor puede actuar solo o en asociación con el receptor T1R2. En asociación los receptores T1R2 y T1R3 responden a todos los azucares o endulzantes. ${ }^{2}$

El sabor umami es provocado solo por dos aminoácidos, el glutamato monosódico y el aspartato; los receptores que utiliza son de tipo T1R1 que están asociados al receptor T1R3, ambos acoplados a la proteína G. ${ }^{2,9} \mathrm{El}$ receptor T1R3 también está asociado al sabor dulce, pues les da el carácter delicioso a estos alimentos. ${ }^{2}$ El glutamato monosódico y la inosina 5'-monofosfato, en pequeñas cantidades, en sinergia provocan un aumento de la percepción de sabor umami; los alimentos que provocan este sabor se encuentran en las carnes, pescados y muchas verduras; ${ }^{8,9}$ por otro lado, el sabor umami contribuye a la detección y reconocimiento de fuentes de proteínas, se ha reportado que en animales suele ser un indicador de una dieta normal en proteínas. . $, 8,11^{-11}$

Recientemente se han realizado diversos estudios sobre el gusto por las grasas, cuyos receptores son CD36 (cluster of differentiation 36) y GPR120 / 40 (receptores de protenina G 120), que se expresan en las células de las papilas gustativas circunvaladas, papilas fungiformes y foliadas, que han permitido develar que no solo la detección de las grasas era propia del sentido del olfato, sino que el sentido del gusto juega un papel muy importante en la detección de cadenas largas de ácidos grasos. La activación de FFA4 o GPR120 central puede reducir el apetito, inhibiendo a las neuronas del neuropéptido, y suprimir los efectos gratificantes de la comida sabrosa, además de su intervención en la sensibilidad a la insulina y la regulación de la inflamación. ${ }^{12,13}$ La detección deficiente de grasa en las células gustativas está asociada a lipotoxicidad inducida por obesidad, diabetes, hipertensión arterial, ateroesclerosis, etc. ${ }^{14}$

\section{Los trastornos del gusto}

La pérdida del gusto puede crear situaciones graves de salud. Una distorsión del gusto puede ser una causa de riesgo para enfermedades que precisen de una dieta específica, como son las enfermedades del corazón, los ataques al cerebro, diabetes y otras más. Al alterarse el sentido del gusto, las personas pueden variar sus hábitos alimenticios; a este trastorno se le conoce como hipogeusia, ageusia cuando no se percibe sabor alguno y disgeusia cuando se percibe continuamente un sabor; salado, rancio, o metálico. En ocasiones, la disgeusia se manifiesta en el síndrome de la boca ardiente. ${ }^{1}$ Las afecciones del gusto se deben a diversas causas, pudiendo darse en los centros nerviosos o lengua propiamente, debido a un desorden en la estructura salival, distorsión en el sentido del olfato y alteraciones psíquico-nerviosas. Se sabe que hay una estrecha conexión de las percepciones olfativas con las sensaciones gustativas. ${ }^{15}$

En el sistema nervioso, los trastornos del gusto se deben a una alteración de la cuerda del tímpano, casi siempre acompañada de parálisis facial periférica y ageusia; en el caso de lesiones del nervio lingual el paciente pierde la sensibilidad motora y gustativa afectando a la lengua en sus dos tercios anteriores; por otro lado, cuando se presenta una lesión del nervio glosofaríngeo, epilepsia, neurosis o psicosis, todas estas 
podrían provocar una sensación gustativa negativa hasta llegar a alguno de los trastornos gustativos. Las lesiones de la boca como estomatitis, glositis, estados saburrales, síndrome de Sjögren, radiación, dentadura artificial, lesiones dentarias y neoplasias, presentan disgeusia o ageusia, igualmente los procedimientos e infecciones orales. Las lesiones en la nariz, como la congestión nasal, además de los síntomas comunes que desarrolla, como fiebre, anorexia o cefalea, desarrollan anosmia, disgeusia o ageusia. ${ }^{15}$ De igual forma las infecciones de las glándulas salivales, parálisis de Bell, infecciones virales, pólipos nasales, sinusitis, faringitis, faringitis estreptocócica, el exceso del consumo de cigarrillo también provocan alteraciones gustativas; ${ }^{16}$ así mismo, el Alzheimer tiene estrecha relación con la disgeusia. Variedad de fármacos crean trastornos en el sentido del gusto, como los antiepilépticos, antibióticos, benzodiacepinas, antitiroideos antihipertensivos entre otros, ${ }^{15}$ así como, por ejemplo, captopril, litio, procarbazina, rifampicina, griseofulvina, claritromicina, penicilamina y fármacos para el tratamiento del cáncer. ${ }^{16}$ La quimioterapia sistémica induce el sabor y el olor, produciendo cambios provocados por daño citotóxico al dividir las células receptoras del gusto y el olfato, interfiriendo en la cantidad y calidad de la saliva. ${ }^{17}$

Los trastornos del olfato y el gusto, actualmente, han emergido como síntomas neurológicos tempranos de la infección por el 2019-nCoV, MERS-CoV y el SARS-CoV-2. La alteración, reducción y pérdida del gusto son causas esenciales y probable repercusión en hábitos alimenticios. ${ }^{4,18}$ La alteración gustativa es síntoma notable en la infección por $2019-n C o V,{ }^{19}$ en algunos casos no presentan congestión nasal o rinorrea y un pequeño grupo los expone de manera ocasional. ${ }^{20} \mathrm{La}$ ageusia y la anosmia parecen ser una característica clínica frecuente del $2019-n C o V ; 21,22$ esto, debido a que el deterioro del gusto en pacientes infectados tiene relación con las RAAS (sistema renina-angiotensina-aldosterona) y se ha demostrado que regulan la percepción del sabor dulce y salado; ${ }^{23,24}$ además, ACE2 (enzima convertidora de angiotensina II) se expresan en buena cantidad en las células epiteliales humanas de la lengua; ${ }^{25}$ por su parte la angiotensina II (Angll) es el principal mediador de la homeostasis del sodio y los fluidos corporales y modula las sensibilidades del gusto salado y dulce. ${ }^{23}$ Estas preferencias se deben a la señalización de la angiotensina II mediante sus receptores gustativos T1r3 y la sensibilidad a la amilorida, sugiriendo que la Angll tiene como órgano blanco periférico a la lengua, se cree que esta enzima es un potente activador del apetito y el gusto por la sal; por ello, el gusto proporciona información relevante sobre la calidad y el valor nutricional de lo que consumimos antes de la ingestión, estos cambios en la percepción del gusto contribuyen en la acción de Angll. ${ }^{25}$ El 2019-nCoV tiene predilección por los AC2 que se expresan con mayor fuerza en la lengua sobre los demás tejidos circundantes, lo que provoca alteraciones del gusto, además del olfato, y en relación a la alteración del sabor dulce y salado. Parma et al. destacan que las alteraciones al dulce y el salado son las más reportadas por los pacientes infectados por 2019-nCoV, ${ }^{26}$ esto nos corrobora la importancia de esta vía de contagio y el manejo clínico que se le debe dar.

El síndrome de Sjögren es un trastorno inflamatorio crónico con reacciones inmunitarias, infiltración linfocítica de las glándulas salivales y lagrimales y xerostomía, que es causa de la sequedad bucal. Tiene una gravedad oscilante en los pacientes, pues presentan cambios en el gusto y el olfato, fisuras en la lengua y los labios (queilitis angular) y un aumento de las caries dentales..$^{27,28}$

La hipertensión arterial y la percepción del sabor salado se encuentran relacionadas y son de importancia clínica en el tratamiento de estos pacientes, pues corrobora la importancia de un tratamiento nutricional adecuado para retrasar las complicaciones sistémicas de la HTA. La identificación a tiempo de alteraciones del sentido del gusto permitirá decidir el planeamiento que logre que los tratamientos antihipertensivos sean efectivos, perfeccionando la perspectiva en la calidad de vida del paciente hipertenso. ${ }^{29}$ Autores como Kubota et al. ${ }^{30}$ concluyeron que el deterioro del reconocimiento del sabor salado se asoció con la hipertensión enmascarada en las mujeres, recomendando la disminución del sabor salado. Por su parte, Michikawa et al. ${ }^{31}$ realizaron un estudio en una población japonesa y relacionaron la percepción del sabor salado, mediante una tira de sabor impregnada de sal a diferentes concentraciones, con la presión arterial, concluyendo que el 
sabor de la sal está asociado con la presión arterial en las mujeres, pero no en hombres. He et al. estudiaron el receptor GPR120 o FFA4 que actúan como receptores de ácidos grasos libres y en el metabolismo de los lípidos, además de tener mucha relación con la diabetes mellitus gestacional, en este estudio se evidenció que estos receptores de grasa, que también se encuentran en la lengua, tienen niveles altos de ARNm de GPR120 en pacientes gestantes con diabetes mellitus y esto se le atribuye a que el receptor GPR120 protege a las personas de los trastornos lipídicos. En el caso de las pacientes DMG, los niveles eran altos y también el nivel de expresión de GPR120 continuó aumentando durante el embarazo, sugiriendo que estas pacientes puedan ser insensibles al GPR120 corroborado con los perfiles lipídicos alterados. El receptor GPR120 puede tener valor terapéutico para los pacientes con DMG ${ }^{32}$ En otros estudios se ha reportado un aumento de la percepción del umbral del sabor dulce en diabéticos de tipo 1 y 2 en comparación con los no diabéticos; estos resultados muestran que la hiperglucemia induce a la alteración de la percepción del sabor dulce debido a una adaptación de las células gustativas por las altas concentraciones de azúcar en sangre..$^{33}$ El GLP-1 (glucagon-like peptide-1) y su receptor se coexpresan en células sensibles al dulce, y se ha demostrado que median la transmisión del sabor dulce; una disminución de este péptido en la saliva de los diabéticos estaría relacionado con la disminución de la percepción del sabor dulce. La leptina es otra hormona metabólica que podría influir entre el sabor dulce y la diabetes, pues se sabe que una alta concentración de la misma tiene relación con un IMC alto, así mismo suprime la respuesta de azucares y edulcorantes naturales y artificiales sin suprimir las otras respuestas gustativas. ${ }^{33}$ Los receptores T1R2 y T1R3 participan en la regulación de procesos metabólicos del azúcar, homeostasis de la glucosa, insulina y la hormona liberadora de la saciedad; tiene importancia como moléculas terapéuticas. La obesidad se asocia con una reducción significativa de las papilas gustativas y puede desempeñar un papel causal en la obesidad en niños y adolescentes. ${ }^{34}$ En un estudio de Kaufmann ${ }^{35}$ se evidencia la codificación del gusto alterada en sujetos obesos. Cada vez hay más pruebas de que el paladar puede ser el eje fundamental de la génesis de la obesidad.

Las anomalías de gusto y del olfato son muy comunes en pacientes con cáncer; Uí Dhuibhir P. et al. ${ }^{36}$ concluyen que las anomalías del gusto y del olfato en pacientes con cáncer, sin tratamiento previo, son comunes; siendo la anomalía del gusto la más frecuente a la del olfato y la anomalía del olfato la que más afecta la calidad de vida, también observó que el $47 \%$ de pacientes estuvo en riesgo de desnutrición; así mismo, McGettigan N. et al. ${ }^{37}$ concluyeron que las anomalías del gusto y del olfato fueron altamente prevalentes en pacientes con cáncer avanzado. Un factor asociado a las anomalías son los niveles de zinc. Según diferentes autores, el zinc tiene un rol importante en la percepción del gusto, mejorando el estado de su función y las sensaciones de disgeusia. ${ }^{38}$ Diversos estudios sobre la deficiencia de zinc en personas con cáncer, cirrosis alcohólica, insuficiencia renal crónica y enteritis regional, que tuvieron alteraciones en el gusto y que se les ha administrado zinc, mostraron mejoría en los niveles de la percepción del gusto, siendo esta una terapia a tomar en cuenta. ${ }^{39}$

\section{Conclusión}

El gusto forma parte de los signos y síntomas de varias enfermedades sistémicas que pueden constituirse en un síntoma predictivo; para ello, es muy importante que el interrogatorio realizado por el profesional pueda contribuir a la detección temprana de enfermedades sistémicas. Por otro lado, resulta necesaria una mejor comprensión de los mecanismos de transducción de la señal gustativa y un mejor seguimiento de los pacientes afectados. La presente revisión muestra que los trastornos del gusto son un indicador en enfermedades como la diabetes mellitus tipo II, la hipertensión arterial, el Alzheimer, cáncer, entre otros; que producen un impacto psicológico en quienes lo padecen, afectando la calidad de vida de las personas. A nivel de la lengua se han estudiado los receptores que detectan los sabores dulce, salado, agrio, amargo y umami, pero aún estos datos son limitados. Así mismo, no es adecuado establecer zonas exclusivas de 
percepción, las células gustativas están repartidas en toda la lengua y en estructuras como la faringe, en mayor y menor proporción.

Cabe señalar que en el tratamiento de los trastornos del gusto se debe tomar en cuenta la administración del zinc para mejorar dichas alteraciones y ofrecer un tratamiento adecuado basado en un buen interrogatorio, examen y detección de la etiología que nos permitirá un pronóstico favorable.

\section{Referencias}

1. Instituto Nacional de la Sordera y Otros Trastornos de la Comunicación. Trastornos del gusto. Dep salud y Serv humanos los EEUU [Internet]. 2013;1-5. Disponible en: https://www.nidcd.nih.gov/sites/default/ files/Documents/health/smelltaste/spanish/Trastornos_del_gusto.pdf

2. Fuentes, A.; Fresno, M. J.; Santander, H.; Valenzuela, $\bar{S}$.; Gutiérrez, M. F. \& Miralles, R. Sensopercepción gustativa: una revisión. Int. J. Odontostomat., 4(2):161-168, 2010.

3. Pozo C. El olfato y el gusto como marcadores precoces de enfermedad de Alzheimer. 2019; Universidad de Murcia - Grado en Nutrición Humana y Dietética 2018/2019. Tesis de grado. Disponible en: https:// digitum.um.es/digitum/handle/10201/91468

4. Risso D, Drayna D, Morini G. Alteration, reduction and taste loss: Main causes and potential implications on dietary habits [Internet]. Vol. 12, Nutrients. 2020. p. 1-16. DOI: 10.3390 / nu12113284

5. Hernández M, Diaz S. La bioquímica y fisiología del sabor. Rev Educ Bioquímica. 2019;38(4):100-4. Disponible en: https://www.medigraphic.com/cgi-bin/new/resumen.cgi?IDARTICULO=91166

6. Guyton C, Hall J. Tratado de Fisiología Médica. Elsevier. Vol. 13a, Tratado de Fisiología Médica. España; 2016. 2924 p.

7. Barrett K, Barman S, Boitano S. Ganong's review of medical physiology. 23.a ed. México: McGraw Hill; 2010. $714 \mathrm{p}$.

8. Chávez Oseki H, Vega Galina VJ, Sierra Arango D, Ramírez Florentino S, Hernández Miramonte Y. Fisiología del gusto. Oral. 2011; 35(2010):625-31. Disponible en: https://www.medigraphic.com/pdfs/oral/ ora-2010/ora1035a.pdf

9. Roper S, Chaudhari N. Taste buds: cells, signals and synapses. Nat Rev Neurosci. 2017 Aug;18(8):485497. doi: 10.1038/nrn.2017.68.

10. Vandenbeuch A, Kinnamon SC. Is the Amiloride-Sensitive Na+ Channel in Taste Cells Really ENaC? Chem Senses [Internet]. 2020;45(4):233-4. doi: 10.1093 / chemse / bjaa011

11. Fajardo $D$. Análisis sensorial de un alimento con umami, para ser utilizado como alternativa saborizante en la disminución de la ingesta de sodio. Universidad Católica de Córdova; 2014. Tesis http://repositorio. ausjal.org/handle/20.500.12032/446620

12. Im DS. FFA4 (GPR120) as a fatty acid sensor involved in appetite control, insulin sensitivity and inflammation regulation. Mol Aspects Med. 2018 Dec; 64:92-108. doi: 10.1016/j.mam.2017.09.001.

13. López Ortiz N. (2020). Estado del arte del gusto graso. Perspectivas en nutrición humana, Setiembre 2020;22(1), 89-98. doi: 10.17533/udea.penh.v22n1a07

14. Deepankumar S, Karthi M, Vasanth K, Selvakumar S. Insights on modulators in perception of taste modalities: a review. Nutr Res Rev. 2019 Dec;32(2):231-246. DOI: 10.1017 / S0954422419000118

15. AndradeG.Alteraciones del gusto en pacientes hipertensos bajotratamiento con fármacos antihipertensivos que acuden al hospital de atención integral del adulto mayor [Internet]. Universidad Central del Ecuador.

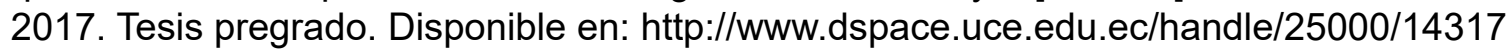

16. MedlinePlus enciclopedia médica. Deterioro del sentido del gusto [Internet]. 2014. 2-5 p. Disponible en: https://medlineplus.gov/spanish/ency/article/003050.htm

17. Van Oort S, Kramer E, de Groot JW, Visser O. Taste alterations and cancer treatment. Current Opinion in Supportive and Palliative Care. 2018 Jun;12(2):162-167. DOI: 10.1097 / spc.0000000000000346

18. Printza A, Constantinidis J. The role of self-reported smell and taste disorders in suspected COVID 19. Eur Arch Otorhinolaryngol. 2020 Sep;277(9):2625-2630. doi: 10.1007/s00405-020-06069-6. 
19. Lechien J, Chiesa-Estomba C, De Siati D y col. Disfunciones olfativas y gustativas como presentación clínica de formas leves a moderadas de la enfermedad por coronavirus (COVID-19): un estudio europeo multicéntrico. Eur Arch Otorhinolaryngol 277, 2251-2261 (2020). Disponible en: https://doi.org/10.1007/ s00405-020-05965-1

20. Barón J, Santiago C, Goizueta G AR y FR. Afectación del sentido del olfato y el gusto en la enfermedad leve por coronavirus (COVID-19) en pacientes españoles. 2020;35(9):633-8. https://doi.org/10.1016/j. nrl.2020.07.006

21. Vaira LA, Salzano G, Fois AG, Piombino P, De Riu G. Potential pathogenesis of ageusia and anosmia in COVID-19 patients. Int Forum Allergy Rhinol. 2020 Sep;10(9):1103-1104. doi: 10.1002/alr.22593. Epub 2020 Jun 15. PMID: 32342636; PMCID: PMC7267531.

22. Cral W, Michels M, Souza C, Queluz D. Dysgeusia and COVID-19: the Importance of the Dentist in the Diagnostic. Int J Odontostomat. 2021;15(1):2-3.

23. Shigemura N, Iwata S, Yasumatsu K, Ohkuri T, Horio N, Sanematsu K, Yoshida R, Margolskee RF, Ninomiya Y. Angiotensin II modulates salty and sweet taste sensitivities. J Neurosci. 2013 Apr 10;33(15):6267-77. DOI: 10.1523 / JNEUROSCI.5599-12.2013

24. Luchiari HR, Giordano RJ, Sidman RL, Pasqualini R, Arap W. Does the RAAS play a role in loss of taste and smell during COVID-19 infections? Pharmacogenomics Journal. 2020 Jan 1. https://doi.org/10.1038/ s41397-020-00202-8

25. Xu, H., Zhong, L., Deng, J. et al. High expression of ACE2 receptor of 2019-nCoV on the epithelial cells of oral mucosa. Int J Oral Sci 12, 8 (2020) 12(1):1-5. Disponible en: https://doi.org/10.1038/s41368-0200074-x

26. Parma V, Ohla K, Veldhuizen MG, Niv MY, Kelly CE, Bakke AJ, et al. More than smell - COVID-19 is associated with severe impairment of smell, taste, and chemesthesis. Chem Senses. 2020;45(7):609-22. doi: 10.1093/chemse/bjaa041

27. López R, Fernández M y HG. Afectación oral en el paciente con síndrome de Sjögren primario. Reumatol clínica [Internet]. 2015;11(6):387-9. http://dx.doi.org/10.1016/j.reuma.2015.03.010

28. Reyes HDL. Manifestaciones orales de las enfermedades sistémicas. Reflexión médica y revisión bibliográfica. Acta Med Cent. 2016;10(1):68-74.

29. López N, Contreras M, Ramírez R. Estado nutricional, presión arterial y su relación con la disgeusia en adultos mayores con hipertensión arterial sistémica de Aguascalientes, México. LUXMED [Internet]. 12 de febrero de 2020];15(43):25-33. DOI: https://doi.org/10.33064/43lm20202497

30. Kubota Y, Higashiyama A, Sugiyama D, Nishida Y, Kubo S, Hirata T, Kadota A, Miyamatsu N, Wakabayashi I, Miyamoto Y, Okamura T. Association between impairment of salty taste recognition and masked hypertension based on home blood pressure in Japanese residents: the KOBE study. Hypertens Res. 2018 Sep;41(9):756-762. doi: 10.1038/s41440-018-0074-9

31. Michikawa T, Nishiwaki Y, Okamura T, Asakura K, Nakano M, Takebayashi T. The taste of salt measured by a simple test and blood pressure in Japanese women and men. Hypertens Res. Mayo de 2009;32(5):399403. doi: $10.1038 / \mathrm{hr} .2009 .31$

32. He Q, Zhu S, Lin M, Yang Q, Wei L, Zhang J, et al. Increased GPR120 level is associated with gestational diabetes mellitus. Biochem Biophys Res Commun. 2019;512(2):196-201. doi: 10.1016/j.bbrc.2019.03.034

33. Neiers F, Canivenc-Lavier MC, Briand L. What Does Diabetes “Taste” Like? Curr Diab Rep. 2016;16(6). https://doi.org/10.1007/s11892-016-0746-2

34. Rohde K, Schamarek I, Blüher M. Consequences of obesity on the sense of taste: Taste buds as treatment targets. Diabetes Metab J. 2020;44(4):509-528. doi: 10.4093/dmj.2020.0058

35. Kaufman A, Kim J, Noel C, Dando R. Taste loss with obesity in mice and men. Int J Obes [Internet]. 2020;44(3):739-43. https://doi.org/10.1038/s41366-019-0429-6

36. Uí Dhuibhir P, Barrett M, O’Donoghue N, Gillham C, El Beltagi N, Walsh D. Self-reported and objective taste and smell evaluation in treatment-naive solid tumour patients. Support Care Cancer [Internet]. 2020 May 5;28(5):2389-96. https://doi.org/10.1007/s00520-019-05017-3 
37. McGettigan N, Dhuibhir PU, Barrett M, Sui J, Balding L, Higgins S, et al. Subjective and objective assessment of taste and smell sensation in advanced cancer. Am J Hosp Palliat Med [Internet]. 2019 Aug 3;36(8):688-96. https://doi.org/10.1177/1049909119832836

38. Heckmann SM, Hujoel P, Habiger S, Friess W, Wichmann M, Heckmann JG, et al. Zinc gluconate in the treatment of dysgeusia - A randomized clinical trial. J Dent Res. 2005;84(1):35-8. doi: $10.1177 / 154405910508400105$.

39. Ripamonti C, Zecca E, Brunelli C, Fulfaro F, Villa S, Balzarini A, et al. A randomized, controlled clinical trial to evaluate the effects of zinc sulfate on cancer patients with taste alterations caused by head and neck irradiation. Cancer. 1998;82(10):1938-45. DOI: 10.1002 / (sici) 1097-0142 (19980515) 82:10<1938 :: aid-cncr18> 3.0.co; $2-\mathrm{u}$

- Conflicto de intereses: La presente investigación no presenta conflicto de intereses entre los investigadores.

- Fuente de financiamiento: La presente investigación fue financiada por los investigadores. 